\title{
AVALIAÇÃO POR SAXS E DSC DAS INTERAÇÕES ENTRE $\mathrm{H}_{2} O$ E RENEX-100
}

Daniella Dias Palombino de Campos

Instituto de Química, Universidade Estadual de Campinas, CP 6154, 13083-970 Campinas - SP, Brasil

Silvana Navarro Cassu

Divisão de Química, Instituto de Aeronáutica e Espaço, Praça Mal. do Ar Eduardo Gomes, 50, 12228-900 São José dos Campos - SP, Brasil

Renata Batista Rivero Garcia, Hiure Anderson Alves da Silva Queiroz, Rene Francisco Boschi Gonçalves e Elizabete Yoshie Kawachi*

Departamento de Química, Instituto Tecnológico de Aeronáutica, Praça Mal. do Ar Eduardo Gomes, 50, 12228-900 São José dos Campos - SP, Brasil

Recebido em 18/5/11; aceito em 16/8/11; publicado na web em 26/9/11

SAXS AND DSC EVALUATION OF INTERACTIONS BETWEEN $\mathrm{H}_{2} \mathrm{O}$ AND RENEX-100. The aggregation behavior of the non-ionic surfactant Renex-100 in aqueous solutions and mesophases was evaluated by SAXS in a wide range of concentrations, between 20 and $30^{\circ} \mathrm{C}$. Complementary, water interactions were defined by DSC curves around $0^{\circ} \mathrm{C}$. SAXS showed that the system undergoes the following phase transitions, from diluted to concentrated aqueous solutions: 1) isotropic solution of Renex aggregates; 2) hexagonal mesophase; 3) lamellar mesophase; and 4) isotropic solution. DSC analysis indicated the presence of interfacial water above $70 \mathrm{wt} \%$, which agreed with the segregation of free water to form the structural mesophases observed by SAXS bellow this concentration.

Keywords: interfacial water; non-ionic surfactant; mesophase.

\section{INTRODUÇÃO}

Formulações que usam água como solvente têm conquistado espaço cada vez maior no desenvolvimento de produtos que sejam menos agressivos ao meio ambiente e à saúde humana. Boa parte destes produtos possui em sua formulação pelo menos uma substância tensoativa que irá conferir funções diversas, tais como, emulsionantes, detergentes, espumantes ou antiespumantes, bactericidas, umectantes, emolientes, dispersantes, solubilizantes, entre outros. Apesar de muitas vezes apresentarem comportamento variável e imprevisível, ${ }^{1}$ a avaliação das interações em sistemas binários água-tensoativo pode auxiliar no entendimento dos mecanismos de ação de um tensoativo em uma dada formulação.

Dentre os tensoativos não iônicos, os polietoxilados ainda são amplamente utilizados como agentes emulsificantes e detergentes. Assim como os tensoativos iônicos, eles formam micelas em água acima de uma concentração micelar crítica (CMC). Quando em concentrações superiores à $\mathrm{CMC}$, as micelas intumescem e se agregam para formar mesofases ordenadas ou cristais líquidos liotrópicos. ${ }^{2}$ A mesofase a ser formada depende de parâmetros físico-químicos, como concentração e temperatura, assim como da estrutura química das moléculas de tensoativo que determinará a forma como estas se agregarão e como elas interagirão com a água.

Em sistemas nanoestruturados (como nas mesofases), a água pode ser classificada de forma rudimentar em 4 categorias: ${ }^{3}$ água livre, água intersticial, ${ }^{4}$ água superficial (física ou quimicamente adsorvida) e água bruta (água quimicamente ligada). Estes diferentes "tipos" de águas têm entalpias e temperaturas de fusão distintas, assim como temperaturas de super-resfriamento anomalamente baixas (o que pode ser provocado por uma redução na energia livre e que pode levar a água a permanecer descongelada a temperaturas muito abaixo da temperatura de congelamento). ${ }^{5}$

*e-mail: bete@ita.br
No presente trabalho, um sistema binário composto por um tensoativo não iônico polietoxilado e água foi estudado por DSC e SAXS, avaliando-se as transições de fase em função das interações entre os componentes e a temperatura do sistema.

\section{PARTE EXPERIMENTAL}

Os sistemas avaliados foram preparados a partir da mistura manual de Renex-100 (Oxiteno), um tensoativo não iônico nonilfenol etoxilado contendo em média $\mathrm{n}=10$ grupos etoxilados (Figura 1 ) e água bidestilada. As amostras preparadas foram designadas como R05, R10, R20, R30, R35, R40, R45, R50, R55, R60, R65, R70, R80 e R90, de acordo com a concentração em \% em massa de Renex-100 em cada sistema. As amostras foram estocadas em frascos tampados, permitindo o equilíbrio dos sistemas à temperatura ambiente.<smiles>CCCCCCCCCc1ccc(C(=O)CCO)cc1</smiles>

Figura 1. Fórmula química do Renex, em que n corresponde ao número médio de grupos etoxilados

A concentração micelar crítica foi determinada a partir do método do anel (Du Noüy) em um tensiômetro Fisher 20. As tensões superficiais de soluções aquosas de Renex-100 de concentrações entre $1,3 \times 10^{-6} \mathrm{~mol} \mathrm{~L}^{-1}$ e $1,3 \times 10^{-3} \mathrm{~mol} \mathrm{~L}^{-1}$ foram determinadas em triplicata. Os valores fornecidos pelo tensiômetro foram corrigidos em relação à direção não vertical de atuação das forças de tensão e à forma complexa do líquido suportado pelo anel. ${ }^{6} \mathrm{O}$ fator de correção $(F)$ depende das dimensões do anel e da natureza da interface, sendo determinado a partir da Equação 1, fornecida no manual do equipamento. 


$$
F=0,7250+\sqrt{\frac{0,01452 P}{C^{2}(D-d)}+0,04534-\frac{1,679 r}{R}}
$$

Nesta equação, $P$ é a tensão superficial aparente fornecida pelo tensiômetro, $C$ é a circunferência do anel, $D$ é a massa específica do líquido, d é a massa específica do ar, $r$ é o raio do fio que forma o anel, e $R$ é o raio do anel. $\mathrm{O}$ fator de correção médio determinado experimentalmente para a água foi $0,932 \pm 0,003$.

O tipo de interação da água com o Renex foi avaliado por calorimetria exploratória diferencial (DSC) em um equipamento DSC Q100 da TA Instruments, calibrado com padrão de índio. As amostras na forma de gel R30, R35, R50, R60 e R65 (massas entre 10 e $15 \mathrm{mg}$ ) foram analisadas em duplicata, entre $-30 \mathrm{e}+30^{\circ} \mathrm{C}$, sob fluxo de $50 \mathrm{~mL} \mathrm{~min}^{-1}$ de $\mathrm{N}_{2}$ e razão de aquecimento de $10^{\circ} \mathrm{C} \mathrm{min}^{-1}$. A variação de entalpia de fusão da água $(\Delta \mathrm{H}), \mathrm{em} \mathrm{J} \mathrm{g}^{-1}$, foi determinada integrando o pico endotérmico.

As análises de SAXS foram conduzidas na linha de luz D11ASAXS1 do LNLS, com radiação de comprimento de onda $\lambda=1,488 \AA$. O sinal transmitido foi coletado em um detector marCCD posicionado a $1000 \mathrm{~mm}$ de distância da amostra. Os sistemas na forma líquida ou gel foram injetados por meio de seringa em um porta-amostra de $1 \mathrm{~mm}$ de espessura entre duas folhas de mica. O tempo de exposição das amostras ao feixe, para coleta do sinal, variou entre grupos de amostras: 500 s para as amostras R05, R10, R20, R30 e R35; 900 s para R40, R45, R50 e R55; e 180 s para R60, R65, R70, R80 e R90. A evolução das fases no sistema binário Renex-100/água foi avaliada em toda a faixa de concentração, a $20{ }^{\circ} \mathrm{C}$. A estabilidade térmica das mesofases foi avaliada pelas amostras na forma de gel R35, $\mathrm{R} 40, \mathrm{R} 45, \mathrm{R} 50$ e R55, a $30^{\circ} \mathrm{C}$. Um banho termostatizado foi usado para controlar a temperatura das amostras. Os padrões de SAXS das amostras foram tratados subtraindo-se o sinal de fundo do detector e normalizando-se em função da intensidade do feixe incidente e da absorção da amostra.

\section{RESULTADOS E DISCUSSÃO}

A curva de tensão superficial de soluções aquosas de Renex-100 de diferentes concentrações é apresentada na Figura 2. Observa-se que a tensão superficial diminui bruscamente de $\sim 65$ para $\sim 35$ dyn $\mathrm{cm}^{-1}$, permanecendo praticamente constante neste valor para soluções mais concentradas. O ponto de intersecção das duas seções de reta que se ajustam aos dados fornece o valor de CMC da ordem de $4 \times 10^{-5}$ mol L ${ }^{-1}$, o que equivale à concentração de aproximadamente $0,003 \%$ em massa de Renex-100 em água. Acima deste valor, portanto, as moléculas de Renex se associam para formar micelas.

Para avaliar as interações entre moléculas de água e de tensoativo, análises de DSC foram realizadas em amostras contendo concentra-

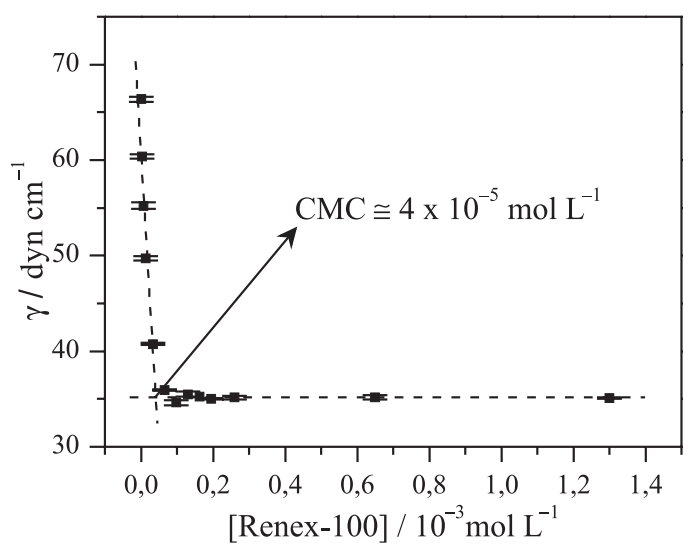

Figura 2. Curva de tensão superficial $(\gamma)$ do Renex-100 em solução aquosa ções de Renex bem acima da CMC, acompanhando a evolução térmica em torno de $0{ }^{\circ} \mathrm{C}$. As curvas de DSC apresentadas na Figura 3 indicam a presença de um evento endotérmico entre $-10 \mathrm{e}+5^{\circ} \mathrm{C}$ para todas as amostras avaliadas, sendo possível observar que a quantidade de calor envolvido nesta transição diminui conforme a quantidade de água na mistura diminui, evidenciando que o evento em questão está relacionado ao processo de fusão da água. Além disso, este evento se desloca para menores valores de temperatura conforme a concentração de Renex aumenta de 30 até $65 \%$, indicando uma maior dificuldade de solidificação da água, provavelmente devido às interações intermoleculares $\mathrm{H}_{2} \mathrm{O}-$ Renex.

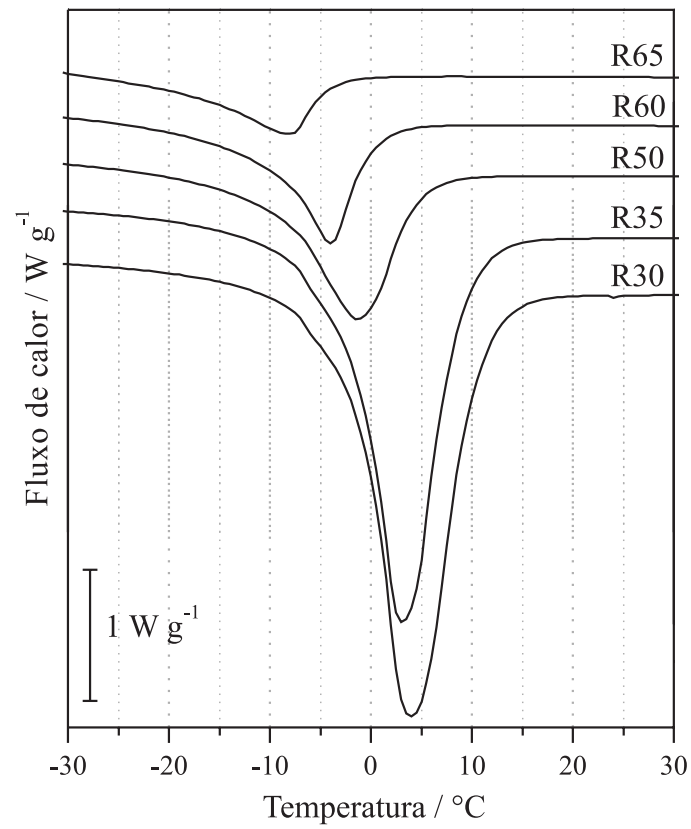

Figura 3. Curvas de DSC para misturas binárias Renex-100/ $\mathrm{H}_{2} \mathrm{O}$ contendo 30, 35, 50, 60 e 65\% em massa de Renex. (Endo $\downarrow$ )

Diferentemente do que se observa em sistemas binários contendo tensoativos iônicos, em que são observados até três eventos endotérmicos que indicam as diferentes interações da água com a porção polar do tensoativo, ${ }^{7}$ apenas um evento endotérmico é observado nas curvas da Figura 3. Para atribuir adequadamente esta transição ao processo de fusão da água, o gráfico de $\Delta \mathrm{H}$ (determinado a partir das curvas de DSC) em função da composição do sistema deve se ajustar a uma reta, como mostra a Figura 4.

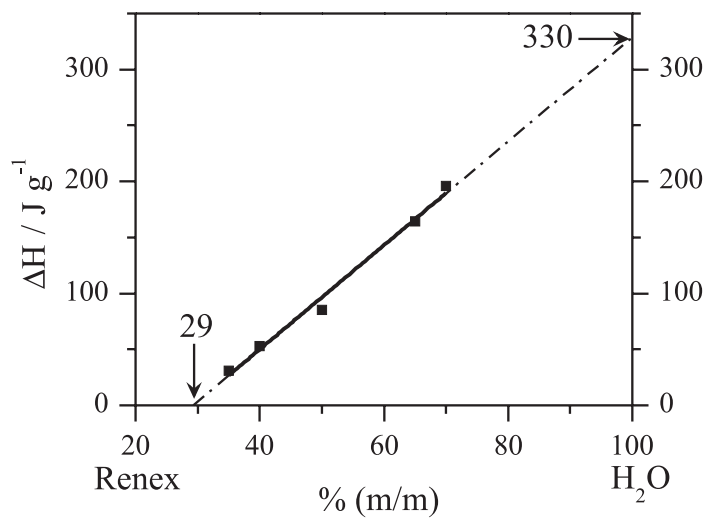

Figura 4. Variação de entalpia dos processos endotérmicos ocorridos em torno de $0^{\circ} \mathrm{C}$. A linha cheia representa o ajuste linear dos dados experimentais (匹) e, a tracejada, a extrapolação do ajuste 
O gráfico da Figura 4 traz duas informações sobre o comportamento da água nestes sistemas binários: a extrapolação do ajuste linear para a composição de $100 \%$ de água fornece o valor de $\Delta \mathrm{H}$ $=330 \mathrm{~J} \mathrm{~g}^{-1}$, resultado próximo ao valor do calor latente de fusão da água reportado na literatura $\left(\Delta \mathrm{H}=334 \mathrm{~J} \mathrm{~g}^{-1}\right),{ }^{8}$ o que indica que o processo endotérmico se refere à fusão da água e não a uma transição para uma mesofase; a extrapolação do ajuste linear para $\Delta \mathrm{H}=0 \mathrm{~J} \mathrm{~g}^{-1}$ indica a quantidade mínima de água necessária para solvatar completamente as moléculas de Renex e, portanto, a quantidade de água que apresenta comportamento de água intersticial. Acima desta concentração, a água adicional passa a apresentar caráter de água livre.

Este resultado foi confirmado pela análise de SAXS de amostras binárias Renex-100/ $\mathrm{H}_{2} \mathrm{O}$ de diferentes composições. O fator estrutura das mesofases pode ser determinado a partir de dados de SAXS com base no padrão de reflexão $\left(\mathrm{q}_{1} / \mathrm{q}_{\mathrm{n}}\right)$ do vetor de espalhamento, q, o qual é definido por $q=4 \pi \operatorname{sen} \theta / \lambda$, sendo que o ângulo de espalhamento é dado por $2 \theta$ e $\lambda$ é o comprimento de onda da radiação. Para mesofases liotrópicas, a posição e as intensidades relativas dos picos de Bragg permitem a determinação das simetrias cristalográficas, por análise das distâncias relativas dos picos, as quais são ditadas pelas leis de reflexão e correspondem à razão entre o parâmetro unitário de cela $(a)$ e as distâncias interplanares (d) para cada mesofase. Para as fases lamelares, os valores de $\mathrm{q}$ dos picos de Bragg apresentam as relações 1:2:3:4:5:... Já para fases hexagonais, a relação entre os picos de Bragg é dada por $1: \sqrt{3}: 2: \sqrt{7}: 3: \sqrt{12}: \sqrt{13}: 4: \ldots$ As fases cúbicas apresentam cinco grupos de simetria, cada um sendo caracterizado por relações específicas entre os picos de Bragg. ${ }^{9}$

Para sistemas isotrópicos contendo apenas aglomerados micelares, no entanto, apenas o fator de forma contribui para o sinal de SAXS, não apresentando padrão de reflexão periódico que define uma estrutura organizada, como no caso das mesofases.

Desta forma, pode-se observar na Figura 5 que o sistema binário se apresenta isotrópico de 5 a 30\% em massa de Renex-100. Como estes valores de concentração estão muito acima da CMC determinada anteriormente, estes sistemas isotrópicos são formados por agregados de tensoativos. Segundo a lei de Guinier, o padrão de espalhamento de uma amostra que contém partículas isotrópicas não correlacionadas apresenta um decaimento monotônico que deve ser proporcional ao quadrado do vetor de espalhamento, de modo que um gráfico de $\log \mathrm{I}(\mathrm{q})$ versus $\mathrm{q}^{2}$ deveria apresentar um ajuste linear na região em que $\mathrm{q} \rightarrow 0$, com coeficiente angular dado por $\left(\mathrm{R}_{\mathrm{g}}{ }^{2} / 3\right)$, sendo possível, então, determinar o raio de giro, $\mathrm{R}_{\mathrm{g}}$, da partícula isotrópica. ${ }^{10}$ Para as amostras analisadas, os gráficos de Guinier (resultados não mostrados) não apresentam linearidade característica, apesar dos padrões de espalhamento das amostras contendo 5 e $10 \%$ de Renex-100 apresentarem decaimento monotônico (Figura 5 R05 e R10). Estas observações indicam a ausência de partículas monodispersas nestas amostras, podendo indicar a presença de partículas assimétricas, polidispersas ou agregadas. Já para as amostras contendo 20 e $30 \%$ de Renex-100, os aglomerados apresentam estruturas correlacionadas que não mostram o decaimento característico na região de Guinier, indicando a formação de agregados micelares.

Entre 35 e $70 \%$ de Renex, os padrões de SAXS mostram picos que guardam uma relação de periodicidade entre si, indicando a presença de mesofases. Os parâmetros de rede da microestrutura são representados pela distância entre planos ( $d$, para estruturas lamelares) e pela aresta do hexágono $(a)$ que se relaciona com a distância entre os planos que passam por dois cilindros adjacentes $(d$, para estrutura hexagonal), ${ }^{11}$ como mostra a Figura 6.

A posição do primeiro pico do padrão de $\mathrm{SAXS}, \mathrm{q}_{1}$, é usada para

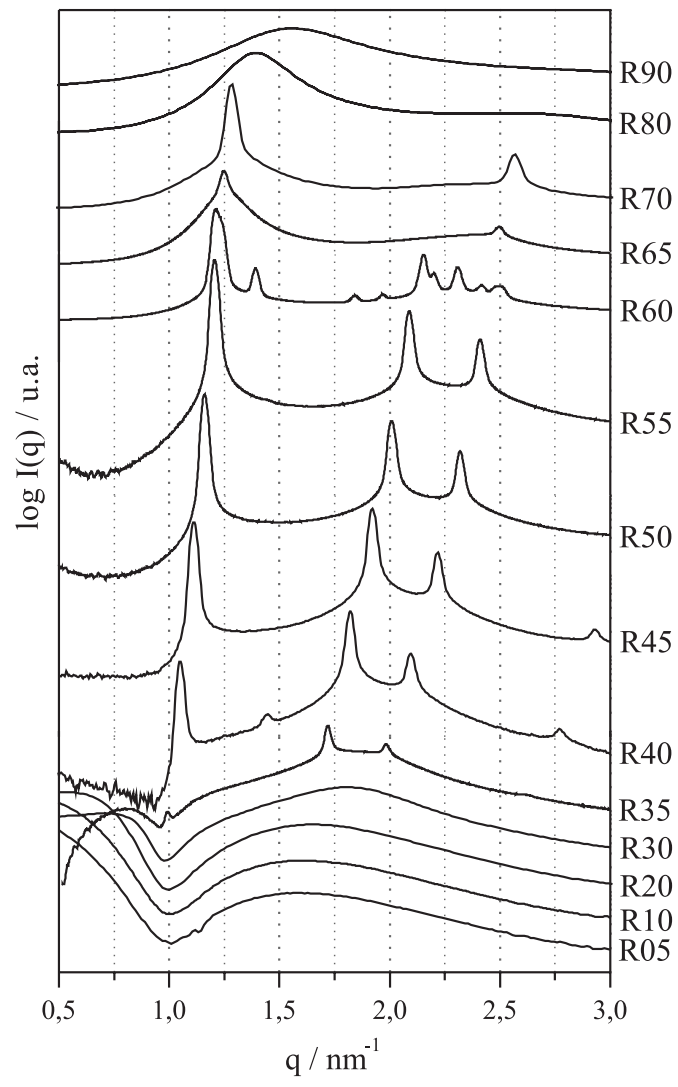

Figura 5. Padrões de SAXS de sistemas binários Renex-100/ $\mathrm{H}_{2} \mathrm{O}$ com diferentes percentuais em massa de Renex, obtidos a $20^{\circ} \mathrm{C}$
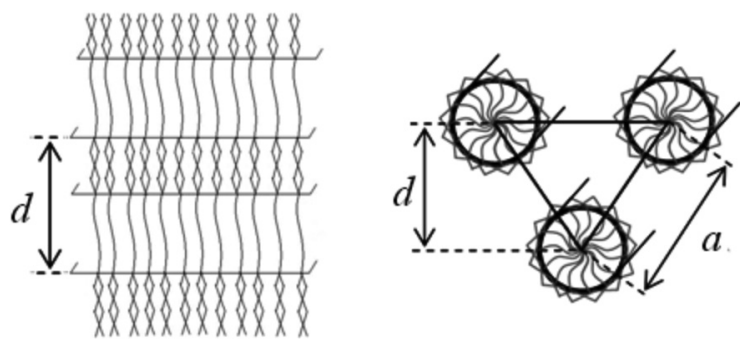

Figura 6. Representação esquemática das mesofases lamelar e hexagonal

avaliar os parâmetros de estrutura das fases lamelar e hexagonal, a partir das Equações 2 e 3, respectivamente: ${ }^{12}$

$$
\begin{gathered}
d=\frac{2 \pi}{q_{1}} \\
d=\frac{2 \pi}{q_{1}}=\frac{\sqrt{3}}{2} a
\end{gathered}
$$

Para as amostras contendo de 35 a 55\% de Renex-100, observase a presença de mesofase hexagonal. Os valores das arestas dos hexágonos e das distâncias interlamelares, determinados a partir da Equação 2 e apresentados na Tabela 1, mostram que ocorre uma compactação destas estruturas conforme a concentração de Renex aumenta no meio, indicando, de forma inversa, que a presença de mais água intumesce o sistema.

A 60\% de Renex em água, forma-se uma estrutura aparentemente mista, de baixa definição, que pode representar uma transição de fase de hexagonal a lamelar. Apesar de não ser possível afirmar com exatidão, a formação de uma fase cúbica justificaria a presença de 
Tabela 1. Parâmetros de estrutura determinados por SAXS para sistemas binários Renex-100/ $\mathrm{H}_{2} \mathrm{O}$

\begin{tabular}{ccccc}
\hline$\%$ Renex & Razão $\mathrm{q}_{1} / \mathrm{q}_{\mathrm{n}}$ & Estrutura & $d / \AA$ & $a / \AA$ \\
\hline$<30$ & --- & $\begin{array}{c}\text { Solução } \\
\text { isotrópica }\end{array}$ & --- & -- \\
35 & $1: \sqrt{3}: \sqrt{4}$ & Hexagonal & 63,5 & 73,3 \\
40 & $1: \sqrt{3}: \sqrt{4}: \sqrt{7}$ & Hexagonal & 59,8 & 69,1 \\
45 & $1: \sqrt{3}: \sqrt{4}: \sqrt{7}$ & Hexagonal & 56,6 & 65,4 \\
50 & $1: \sqrt{3}: \sqrt{4}$ & Hexagonal & 54,2 & 62,5 \\
55 & $1: \sqrt{3}: \sqrt{4}$ & Hexagonal & 52,4 & 60,5 \\
60 & $1: \sqrt{3}: \sqrt{4}$ & Hexagonal & 51,9 & 60,0 \\
& $1: 2: \ldots$ & Lamelar & 51,1 & --- \\
65 & $1: 2: \ldots$ & Lamelar & 50,3 & --- \\
70 & $1: 2: \ldots$ & Lamelar & 49,1 & --- \\
$>80$ & ---- & Solução & --- & \\
& & isotrópica & & \\
\hline
\end{tabular}

alguns picos de baixa intensidade não relacionados às fases hexagonal e/ou lamelar. Entre 65 e $70 \%$, apenas fases lamelares são observadas. A Tabela 1 resume as mesofases identificadas e seus respectivos parâmetros de rede calculados a partir das Equações 2 e 3.

Com base nestas informações, conclui-se que o sistema binário Renex-100/ $\mathrm{H}_{2} \mathrm{O}$ sofre as seguintes transições isotérmicas $\left(20{ }^{\circ} \mathrm{C}\right)$, conforme a concentração de tensoativo aumenta:

isotrópico $\stackrel{35 \%}{\longrightarrow}$ hexagonal $\stackrel{60 \%}{\longrightarrow}$ lamelar $\stackrel{80 \%}{\longrightarrow}$ isotrópico

sendo possível acompanhar a evolução de forma e de estrutura dos agregados de tensoativos, os quais se apresentam inicialmente como partículas não correlacionadas que intumescem e formam cilindros que se agregam em estruturas hexagonais e, posteriormente, se abrem para formação das camadas lamelares.

Acima de $70 \%$ de Renex (ou abaixo de $30 \%$ de água), o sistema perde sua estruturação. De acordo com a curva de variação de entalpia da Figura 3, acima desta concentração de tensoativo, as moléculas de água estão completamente envolvidas com a solvatação dos grupos etoxilados da cabeça polar do Renex, não estando em quantidade suficiente para introduzir uma organização adicional. Tomando por base que o Renex-100 apresenta, em média, 10 grupos etoxilados por molécula, verifica-se que $30 \%$ em água correspondem a aproximadamente 1,5 moléculas de água por átomo de oxigênio presente na cabeça polar deste tensoativo. Nesta situação, portanto, não há água livre no sistema e a adição de mais água deve ser incorporada intersticialmente, de modo a possibilitar a formação de pequenas regiões ricas em água e outras, ricas em tensoativo, formando as mesofases. A inclusão do excesso de água na região interfacial justifica o intumescimento das estruturas hexagonal e lamelar observadas no sistema.

A interação intersticial da água com os grupos etoxilados do tensoativo ocorre por ligações de hidrogênio constituídas entre os átomos de hidrogênio da água (polarizados positivamente) e os átomos de oxigênio dos grupos etoxilados (polarizados negativamente). Como as ligações de hidrogênio costumam envolver energias relativamente baixas e que apresentam valores da ordem de grandeza da energia térmica $\left(k_{B} \mathrm{~T}\right)$, a temperatura do sistema é um parâmetro que deve ser controlado, já que um brando aquecimento pode ser suficiente para aumentar a agitação térmica do sistema e desestabilizar as mesoestruturas, por rompimento das ligações de hidrogênio. Para avaliar a influência da temperatura na estabilidade dos sistemas hexagonais,

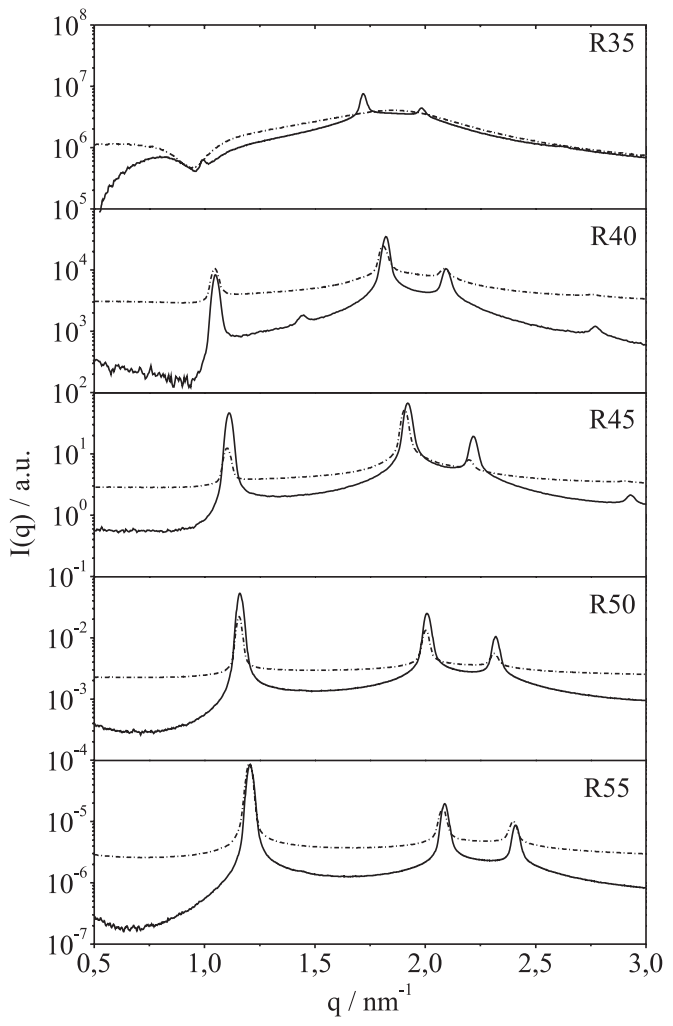

Figura 7. Padrões de SAXS comparativos para amostras binárias Renex-100/ $\mathrm{H}_{2} \mathrm{O}$ a 20 (-) e a $30{ }^{\circ} \mathrm{C}(--)$

SAXS foi conduzido a 20 e a $30{ }^{\circ} \mathrm{C}$ e os resultados são mostrados na Figura 7.

Observa-se que em todas as composições o aumento de temperatura tende a diminuir a intensidade dos picos de espalhamento, indicando uma tendência maior à desestabilização das mesofases a $30^{\circ} \mathrm{C}$. No caso do tensoativo Triton X-100, que possui a mesma estrutura molecular do Renex-100, porém com a parte hidrofóbica constituída de oito átomos de carbono em cadeia ramificada (em contraponto à cadeia linear de nove átomos de carbono do Renex), a literatura ${ }^{1}$ mostra que a temperatura consoluta superior da fase hexagonal está em torno de 30 ${ }^{\circ} \mathrm{C}$. No caso do sistema Renex-100/ $\mathrm{H}_{2} \mathrm{O}$, observa-se uma diminuição na intensidade dos picos, porém a estrutura hexagonal é mantida, incluindo os parâmetros de estrutura $(d \mathrm{e} a)$, indicando que a temperatura consoluta superior deste sistema é maior que $30^{\circ} \mathrm{C}$.

\section{CONCLUSÕES}

A associação das técnicas de DSC e SAXS possibilita a avaliação das interações intermoleculares em sistemas binários água/ tensoativo não iônico Renex-100. Para sistemas contendo mais de $70 \%$ em massa de Renex, as moléculas de água apresentam comportamento de água intersticial e o sistema apresenta-se isotrópico. A incorporação de mais água torna o sistema estruturado, com o excesso comportando-se como água livre e segregando-se para formar estruturas lamelares (de 60 a $70 \%$ de Renex) e hexagonais (de 35 a $60 \%$ de Renex). A mesofase hexagonal é sensível ao aumento de temperatura, porém aquecimento a $30{ }^{\circ} \mathrm{C}$ não chega a desestabilizar totalmente o sistema, indicando que a temperatura consoluta superior desta fase é maior que $30{ }^{\circ} \mathrm{C}$.

\section{AGRADECIMENTOS}

Ao Laboratório Nacional de Luz Síncrotron, CNPEM/MCT, 
por disponibilizar a linha de luz (proposta D11A-SAXS1-8078) e ao $\mathrm{CNPq}$ pelo apoio financeiro (Processo 475971/2007-8). Agradecimento especial ao Prof. C. A. Bertran, do IQ/Unicamp, pelas valiosas discussões.

\section{REFERÊNCIAS}

1. Ahir, S. V.; Petrov, P. G.; Terentjev, E. M.; Langmuir 2002, 18, 9140.

2. Mitchell, D. J.; Tiddy, G. J. T.; Waring, L.; Bostock, T.; McDonald, M. P.; J. Chem. Soc., Faraday Trans. 1983, 79, 975.

3. Schulz, P. C.; J. Therm. Anal. 1998, 51, 135.

4. Senatra, D.; Pratesi, R.; Pieraccini, L.; J. Therm. Anal. 1998, 51, 79.

5. Ezrahi, S.; Aserin, A.; Garti, N. Em Adsorption and aggregation of surfactants in solution; Mittal, K. L.; Shah, D. O., eds.; Marcel Dekker: New York, 2003, cap. 5.
6. Shaw, D. J.; Introduction to colloid and surface chemistry, $4^{\text {th }}$ ed., Butterworth Heinemann: Oxford, 1992.

7. Casillas, N.; Puig, J. E.; Olayo, R.; Hart, T. J.; Franses, E. I.; Langmuir 1989, $5,384$.

8. Em Handbook of Chemistry and Physics; Lide, D. R., ed.; 82 $2^{\text {nd }}$ ed., CRC Press: Boca Raton, 2000.

9. Yaghmur, A.; Campo, L.; Salentinig, S.; Sagalowicz, L.; Leser, M. E.; Glatter, O.; Langmuir 2006, 22, 517.

10. Stribeck, N.; X-ray scattering of soft matter, Springer-Verlag: Heidelberg, 2007.

11. Borsali, R.; Pecora, R.; Soft matter: scattering, imaging and manipulation, $4^{\text {th }}$ ed., Springer-Verlag: Heidelberg, 2008, vol. 2.

12. Soni, S. S.; Brotons, G.; Bellour, M.; Narayanan, T.; Gibaud, A.; J. Phys. Chem. B 2006, 110, 15157. 